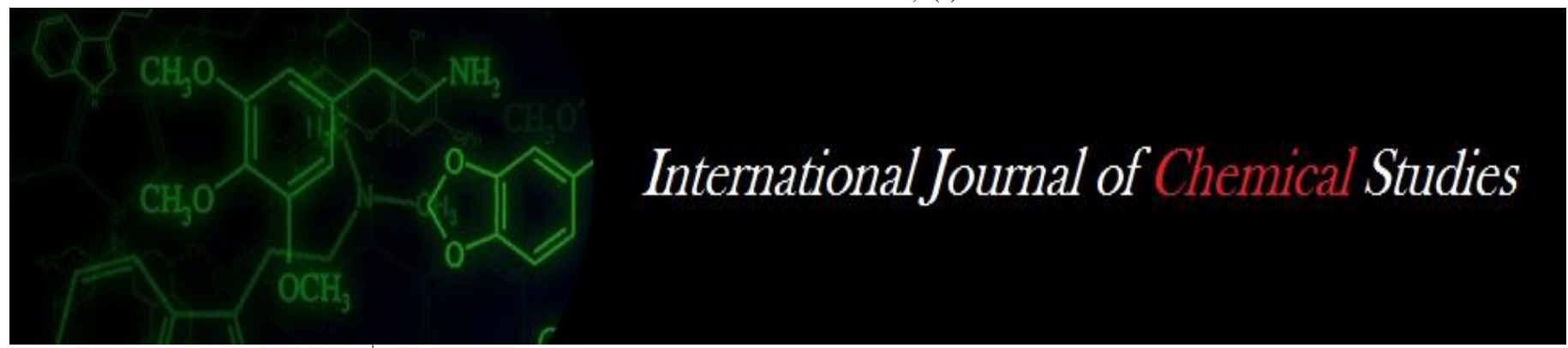

P-ISSN: 2349-8528

E-ISSN: 2321-4902

www.chemijournal.com

IJCS 2020; 8(3): 2984-2988

(C) 2020 IJCS

Received: 28-03-2020

Accepted: 30-04-2020

V Krishnamoorthy

Horticulture Research Station,

Tamil Nadu Agricultural

University, Thadiyankudisai,

Perumbarai (PO), Batlagundu (via), Dindugal District,

Tamil Nadu, India
Corresponding Author:

V Krishnamoorthy

Horticulture Research Station

Tamil Nadu Agricultural

University, Thadiyankudisai,

Perumbarai (PO), Batlagundu

(via), Dindugal District,

Tamil Nadu, India

\section{Heterosis studies for growth, yield and quality parameters in ridge gourd [Luffa accutangula (Roxb.) L.]}

\section{Krishnamoorthy}

DOI: https://doi.org/10.22271/chemi.2020.v8.i3aq.9672

\begin{abstract}
A field trial carried out at Department of Horticulture, Agriculture College and Research Institute, TNAU, Madurai to study the heterosis for growth, flowering, fruit and quality traits of ridge gourd. Twenty four ridge gourd hybrids, six female parents (lines), four male parents (Testers) and one commercial check were sown in the field during kharif, 2018. The highest standard heterosis value was noticed for days to first male flower in L5XT3 (29.50), days to first female flower in L5XT2 (32.99), L5XT4 (32.99), node to first male flower in L5XT3 (347.37), node to first female flower in L3XT1 (80.51), sex ratio in L3XT1 (-33.54), days to harvest in L3XT3 (-13.20), vine length in L5XT2 (-43.90), fruit length in L5XT1 (56.79), fruit girth in L5XT4, L5XT1 (25.53), fruit rind thickness in L6XT4 (75.00), L3XT2 (50.00), fruit flesh thickness in L6XT3 (-18.92), number of fruits per plant in L3XT2 (178.21), L3XT1 (149.82), yield per plant and yield per hectare in L2XT3 (279.45), L3XT2 (221.92), L3XT1 (204.79). The heterosis for quality parameters found to be high in L2XT2 and L3Xt2 (47.06 and 23.53) for total soluble solids, for dry matter content in L6XT2 (147.83), moisture content in L6XT2 (8.67) and for total crude fiber content in L3XT1 (45.00). The L3XT1 and L3XT2 found to show significant heterosis in the desired direction for most of the traits under study and they can be exploited as desirable hybrids.
\end{abstract}

Keywords: Heterosis, ridge gourd, Luffa accutangula, kharif

\section{Introduction}

Among the cucurbitaceous vegetables ridge gourd (Luffa accutangula) is one of the vegetable which has good dietary fiber. It is commercially cultivated mainly during kharif season in India. The matured dried fruit is used for fiber extraction. Ridge gourd is popularly known as kalitori, angled gourd and angled loofah. It has chromosome number $2 \mathrm{n}=26$. Ridge gourd is popularly consumed as vegetable in Asia, African and Arabic countries. In India it is common vegetable in daily diet (Jyothi et al., 2010) ${ }^{[7]}$. Ridge gourd is also known as vegetable of diet food due its high content of moisture (92\%), low calorific value due to low sugar and low protein content. The fruit contains very low total soluble sugar of $1.5 \mathrm{~g}$, dietary fiber $0.5 \mathrm{~g}$, niacin $0.4 \mathrm{mg}$, vitamin-A $410 \mathrm{IU}$, vitamin C $12 \mathrm{mg}$, potassium 139mg, calcium $20 \mathrm{mg}$, iron $0.36 \mathrm{mg}$, magnesium $14 \mathrm{mg}$ and phosphorous $32 \mathrm{mg}$ in $100 \mathrm{~g}$. It also contains antioxidant compounds such as flavonoids $159 \mathrm{mg}$ and phenolics $0.74 \mathrm{mg}$ (Kandoliya et al., 2016) ${ }^{[8]}$. Ridge gourd has been also used extensively in Indian traditional system of medicine as diuretic, expectorant, laxative, purgative, hypoglycemic agent and bitter tonic. Ridge gourd has sweet taste after cooking, cooling in nature and easy to digest. They form a low calorie diet, which is considered good for diabetes. Both the soft pulp and skin of ridge gourd are used in various recipes, especially in south Indian cuisine. Chutneys made from the the peel of ridge gourd is known for their health benefits (Pullaiah, 2006) ${ }^{[14]}$. It is reported to contain many phytochemicals such as flavonoids, saponins, luffangulin, sapogenin, oleanolic acid and cucurbitacin B. In India the production of ridge gourd has to be increased to meet the increased demand. To increase the productivity new varieties and hybrids has to be evolved. Presently few varieties are available in India. Hence, the present study was takenup to develop hybrids which gives higher yield. The objective of this study was to estimate heterosis over the existing commercial check to identify high yielding with good quality. 


\section{Materials and Methods}

The experiment was conducted at Department of Horticulture, Agricultural College and Research Institute, Tamil Nadu Agricultural University, Madurai during kharif 2018 in randomized block design with three replication in order to study the heterosis for growth, yield and quality in ridge gourd. Twenty four F1 hybrids and one commercial hybrid as check utilized in the study. The hybrids were obtained from the cross between six female parents viz. L1 (PKM-1), L2 (CO1), L3 (Virdhunagar local), L4 (Seranmadevi Local), L5 (Arka Sujath), L6 (Arka sumeet) and four male parents viz. T1 (Periyakottai Local), T2 (Alathur Local), T3 (Kannapatti Local), T4 (Srirampuaram Local) crossed in Line X Tester matting design. The seeds obtained in each crosses were extracted. The field was prepared by proper and ploughing and Azospirillum, Phosphobacteria at the rate of $2 \mathrm{~kg}$, Pseudomonas fluorescens $2.5 \mathrm{~kg}$ mixed with $50 \mathrm{~kg}$ farm yard manure and neem cake $100 \mathrm{~kg}$ per hectare was applied. The pits size of $30 \mathrm{~cm} \mathrm{X} 30 \mathrm{~cm} \mathrm{X} 30 \mathrm{~cm}$ at $2.5 \mathrm{~m}$ between row and $2 \mathrm{~m}$ between plants in each row were taken. The pits were filled with $10 \mathrm{~kg}$ of farm yard manure, $6 \mathrm{~g}$ of nitrogen, $12 \mathrm{~g}$ of phosphorous, $12 \mathrm{~g}$ of potassium. The seed obtained in each cross was treated with Trichoderma viride at the rate of $4 \mathrm{~g}$ per $\mathrm{kg}$ of seeds. Three seeds were dibbled in one pit, 15 days after sowing seedlings of two per pit was maintained. The recommended plant protection measures were takenup against the pest and disease occurred in the crop. The observations were registered on various parameters are days to first male flower, days to first female flower, node to first male flower, node to first female flower, sex ratio, vine length (m), days to harvest, fruit weight $(\mathrm{g})$, fruit length $(\mathrm{cm})$, fruit girth, rind thickness (mm), flesh thickness ( $\mathrm{mm})$, number of fruits/ plant, yield/plant (g), yield/ha (t/ha), total soluble solids (TSS), dry matter content (\%), moisture content (\%) and total crude fiber $(\mathrm{mg} / 100 \mathrm{~g})$. The data recorded on various traits were subjected to Analysis of variance by following procedure of Gomez and Gomez (1984) ${ }^{[4]}$, estimation on standard heterosis as per the method of Fonseca and Patterson (1968) ${ }^{[2]}$.

\section{Result and Discussion}

The analysis of variance exhibited that the mean squares due hybrids as well as parents were significant for most of the characters (Table1). The vine length, sex ratio, fruit rind thickness, flesh thickness, fruit diameter, total soluble solids, total crude fiber content. It shows the existence of genetic variability among the hybrids and parents used in the experiment. The variance due to parents against hybrids was significant for the most of the traits. Thus parents and hybrids performance was different from each other for days to first male flower, days to first female flower, node to first male flower, node to first female flower, days to harvest, fruit length $(\mathrm{cm})$, fruit weight, no. of fruits/ plant, yield/ha $(\mathrm{t} / \mathrm{ha})$, dry matter content $(\%)$ and over all heterosis presence evident from significance of parent verses hybrid.

The main aim of heterosis breeding is quantum jump in yield of crop plants. Heterosis over commercial check for 19 traits are given in the Ttable 2. The result indicated that the phenomenon of heterosis was of a general occurrence for important characters in the study. Several study reports also showed substantial heterosis for various agronomic characters by Mole et al. (2001) ${ }^{[10]}$, Gautham et al. (2004) ${ }^{[3]}$ and Nandhini et al. (2018) ${ }^{[11]}$.

The significant positive heterosis was ranged from 1.24 to 29.50. The highest heterosis was observed in L5XT3 (29.50), L5xT4 (19.88) and L2XT4 (18.94) for days to first male and female flowers appearance. It is responsible for earliness in the first harvest. Hedau and Sirohi (2004) ${ }^{[5]}$ also reported heterosis over top parent. The node to first male and female flowers appearance significantly maximum heterosis in cross L3XT1 (80.52), L5XT2 (76.62), L5XT3 (75.32), L1XT3 (71.43). Indistinguishable results in water melon was reported by Aravindakumar et al. (2005) ${ }^{[1]}$. The highest heterosis over standard check indicated earliness. The sex ratio is one of the traits for high yield. The present study showed the negative heterosis for sex ratio that is less number of male flowers and more number of female flowers. Fifteen crosses showed negative significant heterosis. Among the fifteen L3XT1 (33.54), L4XT4 (-22.82), L3XT2 (-27.35) showed highest negative heterosis which shows the plant produce more female flower over other cross and check hybrid.

The negative significant heterosis for days to harvest found in three crosses L3XT3 (-13.20), L1XT1 (-8.05) and L3XT2 (7.38) which was earlier than the check hybrid. The positive heterosis was registered inL4XT1 (32.21), L5XT2 (32.21) which was early among the other hybrids. Ram et al. (2004) ${ }^{[15]}$ observed similar results in ridge gourd. The vine length among the hybrids showed that twenty were significantly negative heterosis. The maximum values of heterosis L5XT2 (-43.90), L1XT1 (-39.90), LzXT1 (-38.68) and L5XT1 ($37.80)$.

The heterosis for fruit weight was significantly negative in 23 hybrids indicates that fruits are smaller than the check. The highest negative value was recorded in L4XT4 (-61.86), L5XT1 (-48.45), L4XT2 (48.42), L4XT1 (-45.02). The fruit length heterosis was significantly positive in L5XT1 (56.79), L6XT4 (49.83), L5XT2 (42.00). It indicates that fruit length was larger than standard check. The fruit girth heterosis was significantly negative in twelve hybrids. The maximum heterosis was recorded in L1Xt2 (-31.91), L5XT4 (25.53), L5XT1 (25.53). Singh et al. (2013) ${ }^{[16]}$ reported positive and significant heterosis over better parent in bottle gourd.

The fruit rind thickness positively significant in three hybrids L6XT4 (75.00), L6XT1 (50.70), L3xT2 (50.00) and in the eleven hybrids it was negatively significant (-25.00). Similar heterosis effect was obtained by Yadav and Kumar (2012) ${ }^{[18]}$ in bottle gourd.The fruit flesh thickness was significantly negative in only three hybrids L6XT3 (-18.92), L3XT2 (16.22) and L4XT4 (16.22). Maximum heterosis was reported by Jadhav et al. (2009) in bitter gourd.

The number of fruits per plant was significantly positive in all the 24 hybrids. The maximum heterosis was recorded in L3XT2 (178.21), L2XT2 (169.50), L4XT2 (157.07), L3XT1 (149.82). Pradip et al. (2014) ${ }^{[13]}$ reported significant heterosis in ridge gourd.The heterosis for yield per plant was significantly positive in 23 hybrids. The highest value was found in L2XT3 (279.45), L3XT2 (221.92), L3XT1 (204.79), L2XT4 (202.74). Lodam et al. (2009) ${ }^{\text {9] }}$ obtained significant positive heterosis in ridge gourd. The heterosis for yield per hectare was significantly positive in 23 hybrids.L2XT3, L3XT2, L2Xt2, L3XT1, L2XT4 hybrids were superior (281.10, 253.01, 216.49, 206.19, 203.78) Narasannavar et al. (2018) obtained significant positive heterosis in ridge gourd. The total soluble solids (TSS), dry matter content, moisture content and total crude fiber content in the fruits are quality parameters of ridge gourd for identifying best hybrids. Two hybrids (L2XT2 and L3T2) exhibited significant positive heterosis (47.06 and 23.53) and another ten hybrids exhibited significant negative heterosis. Venugopalareddy et al. (2019) [17] observed positive heterosis in the hybrid of Kulgod Local X SG-3 (12.82). The dry matter content heterosis was positive 
and significant in fourteen hybrids. The high standard heterosis was exhibited in L6XT2 (147.83), L5XT3 (135.74) and L4XT2(109.21).The less moisture content in the fruits is more preparable. The negative significant heterosis is more desirable among the hybrids. The hybrids L6XT2 (-8.67),
L5XT3 (-7.96) and L4XT2 (-6.40). The total crude fiber heterosis was positively significant in all the 24 hybrids. The high level of heterosis occurred in L3XT1 (45.00), L4XT4 (42.50) and L3XT2 (40.00).

Table 1: Analysis of variance for Line $X$ Tester various characters of ridge gourd

\begin{tabular}{|c|c|c|c|c|c|c|c|c|}
\hline S. No. & Character & Replications & Genotypes & Crosses & Lines & Testers & Line X Tester & Error \\
\hline & Degrees of freedom & 2 & 24 & 23 & 5 & 3 & 15 & 24 \\
\hline 1 & Days to first male flower & 4.205 & $11.120 * *$ & $10.489 * *$ & $8.860 *$ & $29.777 * *$ & $7.175 * *$ & $2.571^{*}$ \\
\hline 2 & Days to first female flower & 0.295 & $24.682 * *$ & $21.838 * *$ & $50.440 *$ & $21.631 * *$ & $12.346 * *$ & $5.137 * *$ \\
\hline 3 & Node to first male flower & 0.295 & $15.053 * *$ & $12.867 * *$ & $19.637 *$ & $20.578 * *$ & $9.068 * *$ & $5.137 * *$ \\
\hline 4 & Node to first female flower & 0.295 & $13.273 * *$ & $7.866 * *$ & $15.645 *$ & 1.804 & $6.484 * *$ & $5.137 * *$ \\
\hline 5 & Sex ratio & 0.124 & 0.847 & 0.854 & $1.640 * *$ & 1.610 & 0.439 & 0.060 \\
\hline 6 & Days to harvest & 0.290 & $188.63 * *$ & $189.090 * *$ & $463.29 *$ & $45.460 * *$ & $126.417 * *$ & $5.142 * *$ \\
\hline 7 & Vine length (m) & 0.000 & 1.871 & 1.790 & $3.282 *$ & 1.575 & 1.3360 & 0.004 \\
\hline 8 & Rind thickness $(\mathrm{cm})$ & 0.000 & 0.023 & 0.025 & 0.041 & 0.003 & 0.0240 & 0.002 \\
\hline 9 & Flesh thickness $(\mathrm{cm})$ & 0.003 & 0.316 & 0.323 & 0.298 & 0.761 & 0.244 & 0.059 \\
\hline 10 & Fruit length $(\mathrm{cm})$ & $12.741 *$ & $114.060 * *$ & $118.980 * *$ & $129.550 *$ & $112.990 * *$ & $116.66 * *$ & $3.130 * *$ \\
\hline 11 & Fruit diameter $(\mathrm{cm})$ & 0.001 & 0.498 & 0.495 & 0.278 & 0.265 & 0.614 & 0.059 \\
\hline 12 & Fruit weight $(\mathrm{g})$ & $122.30 * *$ & $5637.80 * *$ & $5468.800 * *$ & $10049.88^{*}$ & $6806.58 * *$ & $3674.26 * *$ & $43.340 * *$ \\
\hline 13 & TSS (brix) & 0.001 & 0.842 & 0.876 & 0.961 & 0.303 & 0.963 & 0.059 \\
\hline 14 & No. of fruits/plant & 9.219 & $13.960 * *$ & $9.000 * *$ & $20.250 *$ & $20.570 * *$ & $2.940 *$ & $3.050 * *$ \\
\hline 15 & Yield (kg/plant) & 0.001 & 1.886 & 1.706 & $3.383 *$ & 1.488 & 1.191 & 0.059 \\
\hline 16 & Yield /ha (tone) & $12.720 *$ & $33.300 * *$ & $29.990 * *$ & $54.210 *$ & $44.150 * *$ & $19.090 * *$ & $3.130 * *$ \\
\hline 17 & Dry matter content $(\%)$ & 0.001 & $16.950 * *$ & $17.480 * *$ & $14.910 *$ & $33.060 * *$ & $15.218 * *$ & 0.059 \\
\hline 18 & Moisture content $(\%)$ & 0.001 & $16.950 * *$ & $17.480 * *$ & $14.910 *$ & $33.060 * *$ & $15.218 * * *$ & 0.059 \\
\hline 19 & Total crude fiber $(\mathrm{mg} / 100 \mathrm{~g})$ & 0.0014 & 0.004 & 0.004 & 0.007 & 0.002 & 0.003 & 0.001 \\
\hline
\end{tabular}

* Significant at $5 \%$ level ** Significant at $1 \%$ level 
Table 2: Estimates of heterosis (\%) over standard check (SV) for various characters in ridge gourd

\begin{tabular}{|c|c|c|c|c|c|c|c|c|c|c|c|c|c|c|c|c|c|c|c|c|}
\hline & & 1 & 2 & 3 & 4 & 5 & 7 & 6 & 12 & 10 & 11 & 8 & 9 & 14 & 18 & 19 & 13 & 15 & 16 & 17 \\
\hline & & $\begin{array}{c}\text { Days to } \\
\text { first } \\
\text { male } \\
\text { flower }\end{array}$ & $\begin{array}{l}\text { Days to } \\
\text { first } \\
\text { female } \\
\text { flower }\end{array}$ & $\begin{array}{c}\text { Node to } \\
\text { first } \\
\text { male } \\
\text { flower }\end{array}$ & $\begin{array}{c}\text { Node to } \\
\text { first } \\
\text { female } \\
\text { flower }\end{array}$ & Sex ratio & $\begin{array}{l}\text { Vine } \\
\text { length } \\
(\mathbf{m})\end{array}$ & $\begin{array}{l}\text { Days to } \\
\text { harvest }\end{array}$ & $\begin{array}{l}\text { Fruit } \\
\text { weight } \\
\text { (g) }\end{array}$ & $\begin{array}{c}\text { Fruit } \\
\text { length } \\
(\mathbf{c m})\end{array}$ & Fruit girth & $\begin{array}{l}\text { Rind thick } \\
\text { ness }(\mathrm{mm})\end{array}$ & $\begin{array}{c}\text { Flesh } \\
\text { thick ness } \\
(\mathbf{m m})\end{array}$ & $\begin{array}{l}\text { No.of } \\
\text { fruits/ } \\
\text { plant }\end{array}$ & $\begin{array}{c}\text { yield / } \\
\text { plant (g) }\end{array}$ & $\begin{array}{c}\text { Yield/ha } \\
\text { (t/ha) }\end{array}$ & $\begin{array}{c}\text { TSS } \\
\text { (brix) }\end{array}$ & $\begin{array}{c}\text { Dry } \\
\text { matter } \\
\text { content } \\
(\%)\end{array}$ & $\begin{array}{c}\text { Moisture } \\
\text { content } \\
(\%)\end{array}$ & $\begin{array}{c}\text { Total } \\
\text { crude fiber } \\
\text { (mg/100g) }\end{array}$ \\
\hline 1 & L1 X T1 & 3.77 & $13.20 *$ & 78.95 & $58.44 * *$ & $-11.15 *$ & $-39.90 * *$ & $-8.05 *$ & $-27.84 * *$ & $4.53 \mathrm{~ns}$ & $-19.15 * *$ & $0.0 \mathrm{~ns}$ & $-13.51 \mathrm{~ns}$ & $103.34 * *$ & $101.37 * *$ & $102.06 * *$ & $5.88 \mathrm{~ns}$ & $64.62 * *$ & $-3.79 * *$ & $12.50 * *$ \\
\hline 2 & L1 X T2 & $15.53 * *$ & $19.80 * *$ & $215.79 * *$ & $61.04 * *$ & $-4.18 \mathrm{~ns}$ & $25.26 * *$ & $1.95 \mathrm{~ns}$ & $-14.09 * *$ & $24.58 * *$ & $-31.91 * *$ & $-25.00 * *$ & $0.00 \mathrm{~ns}$ & $108.50 * *$ & $115.75 * *$ & $146.65 * *$ & $-20.59 * *$ & 31.77 ** & $-1.86 * *$ & $17.50 * *$ \\
\hline 3 & L1 X T3 & $35 *$ & $29 * *$ & \begin{tabular}{|l|l|}
105.26 \\
\end{tabular} & $71.43 * *$ & $-16.29 * *$ & $-5.75 * *$ & $24.29 * *$ & $-15.81 * *$ & 18.47 ** & & & $-2.70 \mathrm{~ns}$ & \begin{tabular}{|l|}
$74.29 * *$ \\
\end{tabular} & $1.37 * *$ & $102.06 * *$ & $-26.47 * *$ & $-21.30 * *$ & $1.25 * *$ & \\
\hline 4 & L1 X T4 & $10.87 *$ & $18.27 * *$ & $184.21 * *$ & $51.95 * *$ & $-19.49 * *$ & $-27.18 * *$ & $22.71 * *$ & $-26.08 * *$ & $-26.83 * *$ & $.51 \mathrm{~ns}$ & $* *$ & $0.00 \mathrm{~ns}$ & \begin{tabular}{|l|}
71.39 \\
$* * *$
\end{tabular} & $.97 * *$ & & $2.94 \mathrm{~ns}$ & $-5.05 \mathrm{~ns}$ & $0.30 \mathrm{~ns}$ & $0 * *$ \\
\hline 5 & L2 X T1 & 5.75 & $13.20 * *$ & 121.05 & $54.55 * *$ & $-17.25 * *$ & $-38.68 * *$ & $30.42 * *$ & $-37.46 * *$ & -16.03* & $-10.64 \mathrm{~ns}$ & $00 \mathrm{~ns}$ & $-13.51 \mathrm{~ns}$ & $126.58 * *$ & $.52 * *$ & \begin{tabular}{|l|}
$95.19 * *$ \\
\end{tabular} & $17.65 *$ & 82.49 ** & $-4.84 * *$ & $27.50 * *$ \\
\hline 6 & L2 X T2 & 3.26 & $14.21 *$ & 78.95 & $66.23 * *$ & $-19.86 * *$ & $-33.62 * *$ & $5.32 \mathrm{~ns}$ & $-14.78 * *$ & $9.95 \mathrm{~ns}$ & $-12.77 \mathrm{~ns}$ & & $-5.41 \mathrm{~ns}$ & $169.50 * *$ & $5.62 * *$ & $216.49 * *$ & $47.06 * *$ & $71.48 * *$ & $-4.19 * *$ & $33.75 * *$ \\
\hline 7 & L2 X T3 & $11.80 *$ & $17.77 * *$ & 5.79 & & $-10.37 *$ & $-30.92 * *$ & 15.3 & $13.40 * *$ & -26.8 & & -25 . & & & & $281.10 * *$ & $2.94 \mathrm{~ns}$ & $24.73 * *$ & & $0 * *$ \\
\hline 8 & L2 X T4 & $94 * *$ & $12.69 *$ & $268.46 * *$ & $58.44 * *$ & $-17.60 * *$ & $-36.41 * *$ & $24.73 * *$ & $\begin{array}{ll}-1.37 \mathrm{~ns} \\
\end{array}$ & $31.71 * *$ & & & 10.8 & $123.67 * *$ & & $203.78 * *$ & $-5.88 \mathrm{~ns}$ & $-58.84 * *$ & $3.45 * *$ & $50 * *$ \\
\hline 9 & L3 X T1 & $3.98 * *$ & $16.75^{* *}$ & $178.95 * *$ & $80.52 * *$ & $-33.54 * *$ & $-36.93 * *$ & $-5.14 \mathrm{~ns}$ & $-10.96 * *$ & $-19.16 * *$ & $-17.02 * *$ & $-25.00 * *$ & -13.5 & $149.82 * *$ & $204.79 * *$ & $206.19 * *$ & $-17.65 *$ & $-29.06 * *$ & $1.70 * *$ & $45.00 * *$ \\
\hline 10 & L3 X T2 & 6.83 & 9.64 & $126.32 *$ & $38.96 *$ & $-27.35 * *$ & $-31.88 * *$ & $-7.38 *$ & $-7.22 * *$ & $2.28 \mathrm{~ns}$ & $89 * *$ & & -16.2 & $1 * *$ & $92 * *$ & $1 * *$ & $23.53 * *$ & $6.86 \mathrm{~ns}$ & $-0.40 \mathrm{~ns}$ & $40.00 * *$ \\
\hline 11 & L3 X T3 & $11.18 *$ & 5.08 & $126.32 *$ & $55.84 * *$ & $-10.71 *$ & $-27.26 * *$ & $-13.20 * *$ & $-21.99 * *$ & -12.54 & $-19.15 * *$ & $-25.00 * *$ & $-37.84 * *$ & $129.48 * *$ & $145.89 * *$ & \begin{tabular}{|l|}
$146.56 * *$ \\
\end{tabular} & $-14.71 \mathrm{~ns}$ & $19.86 * *$ & $-1.16 * *$ & $27.50 * *$ \\
\hline 12 & L3 X T4 & $10.87 *$ & $15.23 * *$ & $147.37 *$ & $35.06 *$ & $-5.49 \mathrm{~ns}$ & $-34.32 * *$ & $2.48 \mathrm{~ns}$ & $-26.12 * *$ & $-33.45 * *$ & $-6.38 \mathrm{~ns}$ & $0.00 \mathrm{~ns}$ & $0.00 \mathrm{~ns}$ & 77.20 ** & $79.45 * *$ & $80.24 *$ & $-23.53 * *$ & $-41.88 * *$ & $2.46 * *$ & $15.00 * *$ \\
\hline 13 & \begin{tabular}{|l} 
L4 X T1 \\
\end{tabular} & 2.48 & 6.09 & & & $-13.59 * *$ & $-30.75 * *$ & $21 * *$ & & & & & & & & & & $-25.99 * *$ & & $5 * *$ \\
\hline 14 & $\mathrm{X}$ T2 & 35 & $28.93 * *$ & 74 & $48.05 * *$ & $-4.18 \mathrm{~ns}$ & $-27.35 * *$ & $12.93 * *$ & $-48.42 * *$ & -1.55 & & & 10.8 & $157.88 * *$ & $8 * *$ & 95.10 & -5.8 & $109.21 * *$ & $-6.40 * *$ & $0 * *$ \\
\hline 15 & \begin{tabular}{|l|} 
L4 X T3 \\
\end{tabular} & 5.59 & $23.35 * *$ & 105.26 & $42.86 * *$ & $8.01 \mathrm{~ns}$ & \begin{tabular}{|l|}
$-35.54 * *$ \\
\end{tabular} & $3.83 \mathrm{~ns}$ & $-17.87 * *$ & \begin{tabular}{|l|}
$-25.78 * *$ \\
\end{tabular} & $-17.02 * *$ & $0.00 \mathrm{~ns}$ & $-21.62 * *$ & $141.10 * *$ & $171.92 * *$ & $172.68 * *$ & $-17.65 *$ & $24.55 * *$ & $-1.44 * *$ & $37.50 * *$ \\
\hline 16 & L4 X T4 & $15.53 * *$ & $17.26 * *$ & $168.42 * *$ & $51.95 * *$ & $22.82 * *$ & $-24.56 * *$ & $4.27 \mathrm{~ns}$ & $-61.86 * *$ & $-37.28 * *$ & $-21.28 * *$ & $-25.00 * *$ & $-16.22 *$ & $106.25 * *$ & $8.22 \mathrm{~ns}$ & $8.25 \mathrm{~ns}$ & $-38.24 * *$ & \begin{tabular}{|l|}
$-11.55 *$ \\
\end{tabular} & $0.68 *$ & $42.50 * *$ \\
\hline 17 & & 1.24 & $2.34 * *$ & & $64.94 * *$ & $-13.24 * *$ & $-37.80 * *$ & $23.25 * *$ & & \begin{tabular}{|l|}
$56.79 * *$ \\
\end{tabular} & & & & & & & $-2.94 \mathrm{~ns}$ & $7.76 \mathrm{~ns}$ & $-0.46 \mathrm{~ns}$ & \\
\hline 18 & L5XT2 & $16.15 * *$ & $32.99 * *$ & 189.47 ** & $76.62 * *$ & $-12.11 * *$ & $-43.90 * *$ & $32.21 * *$ & \begin{tabular}{|l|}
$-2.06 \mathrm{~ns}$ \\
\end{tabular} & 42.00 ** & $17.02 * *$ & $-25.00 * *$ & $-8.11 \mathrm{~ns}$ & 91.07 ** & $122.60 * *$ & \begin{tabular}{|l|}
$153.44 * *$ \\
\end{tabular} & $-11.76 \mathrm{~ns}$ & $20.94 * *$ & $-1.23 * *$ & $17.50 * *$ \\
\hline 19 & L5XT3 & $29.50 * *$ & $28.43 * *$ & $347.37 * *$ & $75.32 * *$ & $-6.10 \mathrm{~ns}$ & $-27.35 * *$ & 20.10 ** & $-15.77 * *$ & $-14.69 *$ & $-17.02 * *$ & $1.25 \mathrm{~ns}$ & $-13.51 \mathrm{~ns}$ & 71.39 ** & $97.95 * *$ & \begin{tabular}{|l|}
$98.63 * *$ \\
\end{tabular} & $-2.94 \mathrm{~ns}$ & $135.74 * *$ & $-7.96 * *$ & $25.00 * *$ \\
\hline 20 & & $19.88 * *$ & $32.49 * *$ & $242.11 * *$ & $58.44 * *$ & & \begin{tabular}{|l|}
$-2.96 *$ \\
\end{tabular} & $12.04 * *$ & & & $-25.53 * *$ & & & & $134.25 * *$ & \begin{tabular}{|l|l|}
$134.88 * *$ \\
\end{tabular} & & \begin{tabular}{|l|}
$64.80 * *$ \\
\end{tabular} & $-3.80 * *$ & $22.50 * *$ \\
\hline 21 & L6XT1 & $1.24 *$ & 4.06 & & $40.26 *$ & $-18.12 * *$ & $18.12 * *$ & & & $-24.74 * *$ & & $5000 * *$ & & $112.06 * *$ & & & $-17.65 *$ & $6.14 \mathrm{~ns}$ & $-0.36 \mathrm{~ns}$ & \\
\hline 22 & L6XT2 & $17.39 * *$ & 10.66 & $126.32 *$ & $49.35 * *$ & $-12.46 * *$ & $12.02 * *$ & $20.26 * *$ & $-35.74 * *$ & $-6.78 \mathrm{~ns}$ & $-4.26 n s$ & $-25.00 * *$ & $0.00 \mathrm{~ns}$ & $152.07 * *$ & $100.00 * *$ & $130.58 * *$ & $-35.29 * *$ & $147.83 * *$ & $-8.67 * *$ & $12.50 * *$ \\
\hline 23 & L6XT3 & $16.15 * *$ & $27.41 * *$ & $194.74 * *$ & $46.75 * *$ & $-3.83 \mathrm{~ns}$ & $-4.36 * *$ & $23.25 * *$ & $-40.21 * *$ & \begin{tabular}{|l|}
$-23.69 * *$ \\
\end{tabular} & $-12.77 *$ & 25.00 *** & $-18.92 * *$ & $132.39 * *$ & $90.41 * *$ & \begin{tabular}{|l|}
$91.41 * *$ \\
\end{tabular} & $5.88 \mathrm{~ns}$ & $34.30 * *$ & $-2.01 * *$ & $17.50 * *$ \\
\hline \multirow[t]{2}{*}{24} & L6XT4 & 17.25 ** & 8.12 & $157.89 *$ & $51.95 * *$ & $0.00 \mathrm{~ns}$ & $0.00 \mathrm{~ns}$ & $20.2 * *$ & $-15.77 * *$ & $49.83^{* *}$ & $-4.26 \mathrm{~ns}$ & 75.00 ** & $-2.70 \mathrm{~ns}$ & $106.25 * * \mid$ & 138.36 ** & $139.18 * *$ & $11.76 \mathrm{~ns}$ & $15.16 * *$ & $-0.89 * *$ & $22.50 * *$ \\
\hline & SE & 1.60 & 2.27 & 2.27 & 2.27 & 0.24 & 0.07 & 2.27 & 6.58 & 1.77 & 0.24 & 0.02 & 0.24 & \begin{tabular}{|l|}
1.75 \\
\end{tabular} & 0.24 & 1.77 & 0.24 & 0.24 & 0.24 & 0.01 \\
\hline
\end{tabular}




\section{Acknowledgement}

We sincerely express thanks to the Division of Vegetable Crops, Indian Institute of Horticulture Research, Bengaluru, India for providing seeds of Arka Sujath and Arka Sumeet.

\section{References}

1. Aravindakumar JS, Prabhakar M, Pitchaimuthu M, Gouda N. Heterosis and combining ability studies in muskmelon (Cucumis melo L.) for earliness and growth parameter. Karnataka Journal of Horticulture. 2005; 1(4):12-19.

2. Fonseca S, Patterson FL. Hybrid vigour in seven parental diallel crosses in common winter wheat (Triticum aestivum L.). Crop Science. 1968; 8:85-88.

3. Gautham B, Neeraja G, Reddy IP. Evaluation of promising hybrids in ridge gourd (Luffa acutangula L.). Journal of Research ANGRAU. 2004; 32:34-38.

4. Gomez KA, Gomez AA. Statistical procedure for agricultural research. John Wiley and sons, Inc. London, UK (2nd edtn), 1984, 13-175.

5. Hedau NK, Sirohi PS. Heterosis studies in ridge gourd. Indian Journal of Horticulture. 2004; 61(3):236-239.

6. Jadhav KA, Garad BV, Kshirsagar DB, Phumal SS, Patil BT, Shinde KG. Heterosis in bitter gourd (Momordica charantia L.).Vegetable Science. 2009; 36(1):143-45.

7. Jyothi V, Ambati S, Asha Jyothi V. The pharmacognostic, phytochemical and pharmacological profile of Luffa accutangula. International Journal of Pharmacy Technology. 2010; 2(4):512-524.

8. Kandoliya UK, Marviya GV, Bodar NP, Bhadja NU, Golakiya BA. Nutritional and antioxidant components of ridge gourd (Luffa accutangula) fruits of promising genotypes and varieties. Scholars Journal of Agriculture and Vetinary Sciences. 2016; 3(5):397-401.

9. Lodam VA, Desai DT, Khandelwal V, Patil PP. Combining ability analysis in ridge gourd. Vegetable Science. 2009; 36(1):113-115.

10. Mole TJ, Dev SN, Rajan S, Sadhankumar PG. Heterosis and combining ability in ridge gourd (Luffa acutangula Roxb.). Vegetable Science. 2001; 28:165-167.

11. Nandhini D, Ananthan M, Krishnamoorthy V, Anand G. Studies on heterosis in ridge gourd [Luffa accutangula (L) Roxb]. International Journal of Current Microbiology and Applied Sciences. 2018; 7(5):3126-3130.

12. Narasannavar A, Devappa V, Fakrudin B, Pitchaimuthu M, Anjanappa M, Sriram S et al. Exploitation of hybrid vigour and combining ability studies for yield and its attributing traits in ridge gourd [Luffa acutangula (Roxb.) L.]. International Journal of Pure and Applied Bioscience. 2018; 6(1):418-425

13. Pradip K, Munshi AD, Behera TK, Sureja AK. Heterosis in ridge gourd (Luffa acutangula Roxb.) using hermaphrodite lines. Indian Journal of Horticulture. 2014; 71(1):61-66.

14. Pullaiah T. Encyclopedia of world medicinal plants. A.P. Regency publications, New Delhi. 2006; 4:1962-1964.

15. Ram D, Mathura Rai, Verma AK, Pandey S. Heterosis and combining ability in sathputia (Luffa hermaphrodita). Vegetable Science. 2004; 31(2):129-134.

16. Singh AK, Pan R, Bhavana P. Heterosis and combining ability analysis in bittergourd (Momordica charantia L.). The Bioscan. 2013; 8(4):1533-1536.

17. Venugopala Reddy M, Patil MG, Kurubar AR, Utpal Das, Shekharagouda Patil. Heterosis studies in sponge gourd for Earliness and qualitative traits. Journal of
Pharmacognosy and Phytochemistry. 2019; 8(1):21322138.

18. Yadav YC, Kumar S. Estimation of heterosis for yield and yield contributing traits in bottle gourd [Lagenaria siceraria (Molina) Standl.]. The Asian Journal of Horticulture. 2012; 7(2):310-314. 